\title{
Le changement climatique dans la région des Grands Lacs
}

Alexandre Taithe

\section{Q OpenEdition \\ 12 Journals}

\section{Édition électronique}

URL : https://journals.openedition.org/eastafrica/383

DOI : $10.4000 /$ eastafrica.383

ISSN : 2790-1076

\section{Éditeur}

IFRA - Institut Français de Recherche en Afrique

Édition imprimée

Date de publication : 1 avril 2014

Pagination : $37-50$

ISSN : 2071-7245

\section{Référence électronique}

Alexandre Taithe, "Le changement climatique dans la région des Grands Lacs », Les Cahiers d'Afrique de l'Est / The East African Review [En ligne], 48 | 2014, mis en ligne le 07 mai 2019, consulté le 09 décembre 2021. URL : http://journals.openedition.org/eastafrica/383 ; DOI : https://doi.org/10.4000/ eastafrica.383 


\section{Le changement climatique dans la région des Grands Lacs}

Alexandre Taithe

\section{Introduction}

« Le changement climatique est une agression des pays riches sur les pays du sud $»^{1}$, déclarait en 2007 Yoweri Museveni à l'occasion d'un sommet de l'Union Africaine. Le Président ougandais résumait ainsi plusieurs points qui reflètent la perception de l'enjeu climatique par de nombreux pays en développement ou parmi les moins avancés. Cette formule fait tout d'abord référence à la responsabilité des États industriels dans les émissions passées de Gaz à Effet de Serre (GES). Corollaire de la responsabilité, la réparation des dommages est ensuite, depuis les négociations Climat de Copenhague ${ }^{2}$ en 2009, au centre des revendications des pays du G77, comme elle 1'était encore au Sommet Climat de Varsovie en novembre $2013^{3}$. Le terme « agression » souligne également à quel point les changements climatiques sont perçus comme une menace potentielle au développement, voire à la sécurité, des États ayant les capacités d'adaptation les plus réduites pour y faire face.

Les pays des Grands Lacs, avec le Kenya et la Tanzanie, ont toujours été des acteurs visibles des différentes étapes des négociations climatiques depuis le Sommet de Bali en 2007, à l'image de la demande, portée par les Présidents Kibaki et Museveni à Copenhague en 2009, de compensations du préjudice subi par les pays les plus pauvres, de même que la motion kenyane lors des Négociations de Doha en 2012 pour la création effective du Fonds Vert pour le Climat, ou encore l'appel lancé par l'Ouganda contre la déforestation lors de la Conférence de Durban en 2011... L'environnement étant encore largement perçu comme un enjeu de coopération de basse intensité, il favorise, il est vrai, des prises de position forte, et n'induit pas ou peu d'effets négatifs en termes d'image, de relations bilatérales, ou même de politique interne.

Cette visibilité dans les arènes internationales sur le climat traduit cependant une véritable crainte de ces États envers un phénomène global aux effets différenciés, d'autant que les modélisations climatiques divergent quant aux bénéfices potentiels et impacts néga tifs tifs du réchauffement en Afrique de l'Est et en République Démocratique du Congo (RDC). Au regard de l'importance sociale et économique de l'agriculture dans cette aire

1 "Drying up and flooding out", The Economist, 10 mai 2007.

2 15ème Conférence des parties à la Convention Cadre des Nations Unies sur les Changements Climatiques (CCNUCC) ou COP15.

3 19ème Conférence des parties à la CCNUCC ou COP19. 
géographique, les évolutions environnementales, même minimes, pourraient y avoir d'importantes conséquences sur la sécurité alimentaire, et la stabilité sociale et politique. L'Afrique des Grands Lacs et 1'Afrique de l'Est font en effet l'objet d'une abondante littérature grise sur les liens entre la conflictualité et le changement climatique (et plus largement les évolutions de l'environnement).

\section{Le changement climatique dans la région des Grands Lacs}

Les modèles climatiques globaux sont sans doute ce qui existe de plus fiable (avec la démographie) pour se projeter à long terme (jusqu'à 2100), mais leur précision décroît à mesure que l'on restreint le cadre géographique auquel on les applique. Le dernier rapport d'évaluation du GIEC, dont le rapport du premier groupe de travail a été publié en septembre 2013, contribue à une connaissance beaucoup plus fine des ressorts régionaux des évolutions climatiques. La température de surface de l'océan Indien s'avère être le principal facteur des précipitations ${ }^{4}$ en Afrique de l'Est. Mais le régime biannuel de pluie dans cette région est également influencé par des phénomènes cycliques comme ENSO (El Niño Southern Oscillation) et les mouvements de la Zone de convergence intertropicale (ou ZCIT, rencontre des vents chauds des deux hémisphères). Et toute modification de la température de l'océan Indien, des vents, ou des courants a une incidence sur l'humidité de l'air, et sur les précipitations en Afrique de 1'Est.

\section{Tableau 1. Principaux impacts du changement climatique ${ }^{5}$}

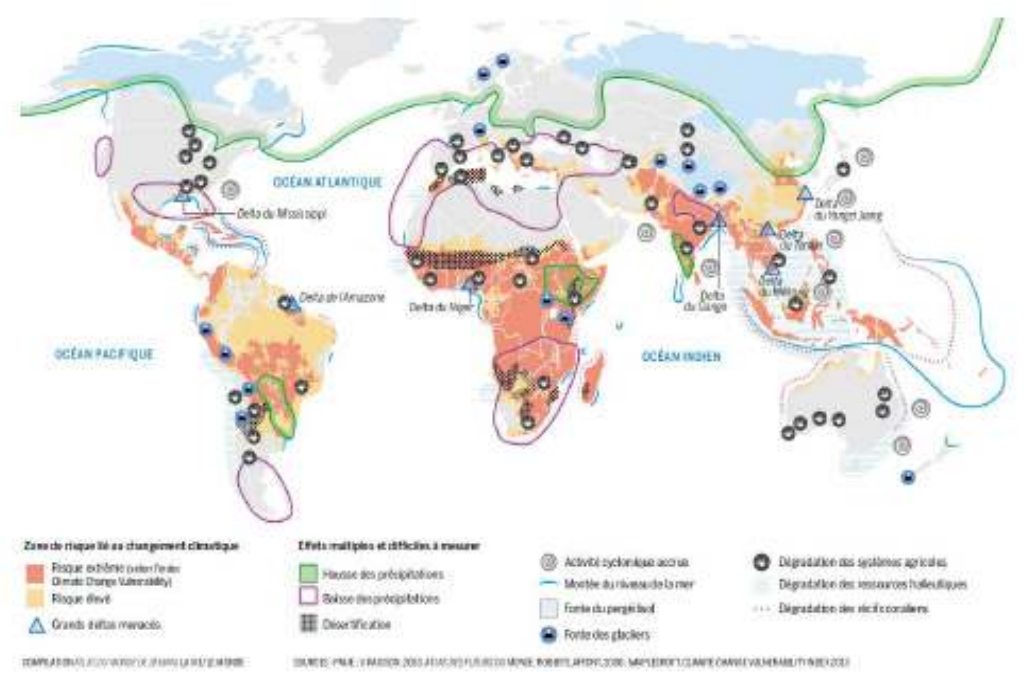

4 IPCC Working Group I, Working Group I Contribution to the IPCC Fifth Assessment Report (AR5), Climate Change 2013: the Physical basis. Chapter 14: Climate Phenomena and their Relevance for Future Regional Climate Change, Genève, 2013, 147p., http://www.climatechange2013.org/ images/uploads/WGIAR5_WGI-12Doc2b_ FinalDraft_Chapter14.pdf

5 «La carte des principaux impacts du réchauffement climatique », Le Monde, 27 septembre 2013, à partir de : Maplecroft, Climate Change and Environmental Risk Atlas 2014. 
L'Afrique de l'Est devrait en moyenne gagner en précipitation et en température, avec une forte variété de situation locale (y compris des volumes de pluie en baisse dans certaines régions). L'exception principale, où l'aridité devrait s'accroître fortement, est une large bande de territoire de part et d'autre de la frontière entre la Tanzanie et le Kenya, partant du littoral puis remontant jusqu'au Soudan, et touchant également la Somalie (cf. tableau $\left.\mathrm{n}^{\circ} 1\right)$.

\section{Impacts du changement climatique sur l'agriculture en Afrique de l'Est et en RDC}

L'international Food Policy Research Institute (IFPRI) a entrepris un travail d'extrapolation de modèles climatiques globaux développés pour le quatrième rapport d'évaluation du GIEC publié en 2007. Ces modèles globaux (CSIRO, MIROC, CNRM, $\mathrm{ECHAM}^{\circ}$ ) ont été transposés à une échelle régionale et nationale. Cela explique une divergence relativement prononcée des résultats, ces modèles n'ayant pas été spécifiquement conçus pour ce changement d'échelle vers le local. De plus, la fiabilité des modèles décroit à mesure que leur résolution spatiale s'affine. La connaissance des facteurs du climat de l'Afrique de l'Est s'est beaucoup améliorée entre le 4ème rapport d'évaluation (2007) du GIEC, et le 5ème (AR5), dont le rapport du groupe 1 (les bases scientifiques du réchauffement), a été publié en septembre 2013. On peut donc espérer des modélisations régionales à la fois plus fines (résolution) et plus fiables dans les prochaines années, à partir du perfectionnement des modèles globaux utilisés pour l'AR5.

Pour projeter des productions agricoles et des demandes en denrées alimentaires, ces modèles ont été croisés par l'IFPRI ${ }^{7}$ avec trois scénarios de développement : optimiste (croissance démographique limitée, forte hausse des revenus par habitant), pessimiste (fort accroissement démographique, faible croissance du revenu par habitant), et un scénario intermédiaire. Les projections climatiques non liées à l'agriculture proviennent d'un scénario unique de développement économique et social, dit « A1B ». Il correspond à une croissance économique rapide, à un pic de population mondiale en 2050 (suivi d'une diminution), et d'un usage équilibré des sources d'énergie (hydrocarbures et non émettrices de Gaz à effet de serre-GES). Le «A1B » est l'un des six scénarios utilisés par le GIEC dans ses précédents rapports d'évaluation du changement climatique, et conduit au 3ème plus grosses émissions de GES. Conçu comme un scénario « Business as usual » à peine vertueux, il apparait aujourd'hui comme optimiste au regard de l'absence de régulation

\footnotetext{
6 Le CNRM est le résultat de la collaboration entre le Centre Européen de Recherche et de Formation Avancée en Calcul Scientifique (CERFAS) et le Centre National de Recherches Météorologiques (CNRM) de Météo France. CSIRO est un modèle climatique développé par l'Australia Commonwealth Scientific and Industrial Research Organisation. ECHAM est une generation de modèles développés par le Max Planck Institute for Meteorology d'Hamburg. MIROC est le 'Model for Interdisciplinary Research on Climate', dévelopé par le Center for Climate System Research de l'Université de Tokyo.

7 Nelson Gerard C., Rosegrant Mark W, et.al, Food security and climate change. Challenges to 2050 and beyond, IFPRI, 2010, 131p., http://www.ifpri.org/sites/ default/files/publications/rr172.pdf
} 
internationale forte des émissions de GES depuis la 15ème Conférence des parties à la Convention Cadre des Nations Unies sur les Changements Climatiques (CCNUCC) tenue en 2009 (COP15 ou « Sommet de Copenhague »).

\section{Tableau 2: Statistiques regionales relatives au secteur agricole $^{8}$}

\begin{tabular}{|c|c|c|c|c|c|}
\hline & $\begin{array}{l}\text { Population } \\
\text { en } 2010 \\
\text { (milions) }\end{array}$ & $\begin{array}{l}\text { Part de } \\
\text { Pagriculture } \\
\text { dans le PIB } \\
2009-2011\end{array}$ & $\begin{array}{l}\text { Population } \\
\text { agricolee, } \\
2010\end{array}$ & $\begin{array}{c}\text { Population } \\
\text { rurale (\%) } \\
2010\end{array}$ & $\begin{array}{l}\text { Part de la } \\
\text { population active } \\
\text { agricole dans la } \\
\text { population active } \\
\text { totale) } 2010\end{array}$ \\
\hline Burundi & 8.4 & 35.2 & 26.8 & 89 & - \\
\hline Kenya & 40.5 & 23.1 & 70.6 & 77.8 & 61.1 \\
\hline Ouganda & 33.4 & 23.4 & 73.5 & 86.7 & 65.6 \\
\hline Rwanda & 10.6 & 32.2 & 89.4 & 81.1 & 78.8 \\
\hline RDC & 66 & 42.9 & 57.2 & 64.8 & - \\
\hline Tanzanie & 44.8 & 27.1 & 73.3 & 73.6 & 76.5 \\
\hline Éthiopie & 82.9 & 41.9 & 77.3 & 82.4 & 79.3 \\
\hline
\end{tabular}

\section{Burundi}

Si le Burundi reçoit globalement des précipitations abondantes (deux saisons des pluies, complétées par une quinzaine de jours de pluie en janvier), la faible proportion de terre arable (un peu plus d'un tiers du territoire) et l'importance d'une culture (la banane) dans la richesse agricole produite constituent des vulnérabilités potentielles au changement climatique. A cela s'ajoutent des différences de précipitations d'un facteur 2 entre les hautes terres et les basses terres (respectivement $2000 \mathrm{~mm}$ par an contre $1000 \mathrm{~mm}$ par an). Plusieurs conséquences du réchauffement climatique dans la zone sont déjà notables : la dégradation des sols et des zones marécageuses, et, avec un moindre indice de fiabilité, la baisse de niveau du lac Tanganyika.

Source largement majoritaire des recettes nationales en devises, le secteur agricole est limité par l'étendue des surfaces arables. Or les scénarios de l'IFPRI prévoient une diminution importante de l'aire sous culture (moins 25\% à 1'horizon 2050), qui pourrait cependant être compensée par des gains de productivité (maïs, manioc). La déforestation s'ajoute aux effets du changement climatique pour expliquer les pertes de terres cultivables.

8 FAO, Statistical Yearbook 2012. Africa. Food and Agriculture, Accra, FAO, 2013, 273p. 
Mais quels que soient les scénarios (de population, de développement, d'impact du changement climatique), le Burundi pourrait avoir à importer du maïs à partir de 2050. La hausse attendue du niveau de vie devrait, même dans les scénarios pessimistes, contribuer à limiter la prévalence de la sous-alimentation, et augmenter le nombre de calories disponibles par jour et par personne malgré l'accroissement démographique.

\section{Rwanda}

Essentiellement sous pluie, l'agriculture rwandaise s'avère sensible aux variations de températures et des régimes de précipitation, d'autant que la part de la population agricole frôle les $90 \%$ de la population totale . La production agricole vivrière est principalement destinée à l'autoconsommation.

Les modèles divergent sur l'évolution des précipitations, certains anticipant un climat plus sec, d'autres prédisant une augmentation (jusqu'à $200 \mathrm{~mm}$ ) des précipitations. Ils convergent en revanche sur un accroissement de la température, de 1 à $2,5^{\circ} \mathrm{C}\left(1,5^{\circ} \mathrm{C}\right.$ maximum pour l'un des modèles).

Ainsi, si la hausse des températures s'accompagne de précipitations plus abondantes, les gains de rendement agricole pourraient être de 25\% dans la moitié Est du Rwanda. A l'inverse, si températures et précipitations ne s'accroissent que modérément, le rendement agricole devrait au contraire diminuer sur tout le territoire (de 5 à $25 \%$ ).

Des températures plus élevées favoriseront l'extension des aires de présence de parasites pour les végétaux et pour l'homme (les vecteurs de la malaria par exemple). Dans le même temps, de nouvelles variétés de plantes agricoles, importantes pour leurs qualités nutritionnelles, pourraient alors cultivées, comme le sorgho. La mise en culture de nouvelles terres deviendrait possible, avec un risque accru de tension entre les aires naturelles protégées et l'extension des zones agricoles. La surface des terres cultivées pourrait ainsi augmenter jusqu'à 50\% d'ici à 2050 (comparé à 2010) pour certaines variétés, comme les pommes de terre. Le déboisement devrait s'accentuer au regard du manque de terres.

\section{Kenya}

Pays au climat aride et semi-aride sur plus de $80 \%$ de son territoire, le Kenya s'avère particulièrement vulnérable au changement climatique. Près de 2 millions de personnes nécessitent une aide alimentaire régulière, ce chiffre pouvant aller jusqu'à 5 millions lors des épisodes de sécheresse. La force de travail agricole ( $61 \%$ de la population active) est surtout composée de petits exploitants. Malgré la baisse continue de la part de l'agriculture dans le PNB, l'importance sociale et économique de l'agriculture pour le Kenya doit contribuer à placer cette dernière au centre des politiques d'adaptation au changement climatique. 
L'évolution des températures exacerbera les caractéristiques climatiques kenyanes : les hausses devraient être les plus prononcées d'ici 2050 dans les parties Est, Nord-Est et côtières du territoire (jusqu'à $+2^{\circ} \mathrm{C}$ ), et plus limitées (entre 0,5 et $1^{\circ} \mathrm{C}$ ) dans les zones à fort potentiel agricole.

Des gains de terres arables sont projetés, notamment dans les plateaux centraux, au Nord du mont Kenya, qui compenseront les pertes probables à proximité de la frontière ougandaise au Nord du lac Victoria. Quel que soit le niveau de développement économique retenu, la production de maïs devrait augmenter, principalement grâce à des gains de productivité. Mais la disponibilité de nourriture (exprimée en kilocalories), ne devrait pas décoller avant 2030 dans le scénario optimiste de développement (population, économie), voire pourrait même reculer dans l'hypothèse d'une forte croissance démographique et d'un développement économique limité. Le cours des produits de base comme le maïs doublerait d'ici à 2050, là où le revenu médian ne gagnerait que 50\% entre 2000 et 2050 .

\section{Ouganda}

Les dernières décennies en Ouganda ont été caractérisées par une variabilité climatique accrue, signe des changements climatiques en cours et confortant la probabilité d'occurrence d'évènements climatiques extrêmes', tels les sécheresses, les inondations ou glissements de terrains.

L'accroissement démographique apparait comme l'une des principales vulnérabilités de l'Ouganda au changement climatique, auquel on peut ajouter la forte part de la population rurale et de la population active agricole dans la population totale.

Les modélisations du climat ougandais en 2050 divergent dans l'absolu, même si elles n'annoncent pas de changements radicaux, d'autant que les précipitations devraient au pire stagner, voire augmenter sur la plupart du territoire. En revanche, le régime des précipitations risque de se modifier, avec des pluies plus intenses, plus concentrées, favorisant le ruissellement et les inondations. Ainsi, malgré un volume de précipitation stable ou en croissance, le nombre de jours sans pluie devrait croitre, signe d'un risque de sécheresse augmenté. Les modèles décrivent des pluies en augmentation pendant les mois les plus chauds, période de croissance des cultures, même dans l'unique cas (modèle CRNM) où globalement, la pluviométrie annuelle décroitrait.

La hausse des températures devrait être située entre +1 et $+2{ }^{\circ} \mathrm{C}$ (fonte accrue des glaces et neiges, évaporation plus forte). Or la couverture glaciaire dans la chaine du Rwenzori est déjà en retrait marqué (moins 40\% entre 1955 et 2008) ${ }^{10}$, conduisant indirectement à la réduction des débits d'étiage. La pression sur les ressources en eau

\footnotetext{
9 Ministry of Water \& Environment, Uganda, Climate Change: a current future threat to the socio-economic development of Uganda, Policy Brief nº1/2010, June 2010, 4p.

10 Ministry of Water \& Environment, Uganda, 2010, op. cit.
} 
s'accroit, réduisant d'ores et déjà le volume des récoltes. Une température plus élevée (notamment pour les deux modèles qui prévoient une hausse légèrement supérieure à $2^{\circ} \mathrm{C}$ ) favorisera les maladies végétales et animales, ainsi que la prolifération de parasites.

Les rendements pour les principales cultures devraient connaitre une forte hausse entre 2010 et 2050, malgré les changements environnementaux : triplement pour le maïs, plus $80 \%$ pour le manioc. Pour certaines variétés comme le manioc, l'aire mise en culture devrait se réduire, limitant ainsi les bénéfices des gains de productivité. Au final, l'Ouganda pourrait devenir un exportateur net de matières premières agricoles en 2050, ce qui n'empêchera pas la persistance de la faim dans certaines aires géographiques (insuffisance du réseau routier, insécurité, évènements climatiques extrêmes...). La variété de l'alimentation demeurera également un enjeu, plus que la quantité (accès à un volume journalier de calories).

\section{République Démocratique du Congo ${ }^{11}$}

L'agriculture est le principal moyen de subsistance pour $70 \%$ de la population congolaise. La dépendance vitale et économique à ce secteur, auquel s'ajoute l'accroissement démographique (avec le triplement de la population depuis 1960), constituent des facteurs forts de vulnérabilité. Tant que les richesses minières de la RDC (tantale, or, diamant, cuivre, zinc, tungstène, étain...) ne seront pas davantage exploitées dans l'économie légale, la part de l'agriculture dans l'économie de la RDC restera la plus élevée comparée aux autres pays des Grands Lacs et de l'Afrique de l'Est (presque $43 \%)^{12}$.

L'accroissement des températures sera plus marqué que dans les autres pays évoqués, avec une hausse comprise entre 1 et $3^{\circ} \mathrm{C}$ entre 2010 et 2000.

Les modèles divergent sur l'évolution des précipitations, même si une large bande Est du territoire, ainsi que la région de Kinshasa, devraient recevoir moins de pluie.

Les pertes de rendement agricole (2050 vs 2000) se concentreront au Nord de la frontière provinciale du Katanga. A l'inverse des gains de production peuvent être espérés dans les provinces les plus occidentales.

La production de manioc, essentiel à la sécurité alimentaire locale, devrait doubler entre 2010 et 2050, davantage grâce à un meilleur rendement qu'à une extension des surfaces mises en culture pour cette variété. Mais la RDC demeurera un importateur de manioc à cet horizon, tant la demande interne est forte. Pour le maïs, le potentiel d'amélioration des techniques de culture est tel que des gains de rendement de $70 \%$ sont envisageables dans les 15 prochaines années. Mais cela ne suffira pas à répondre aux besoins internes en maïs dès 2025. Ces progrès de production ne feront pas disparaitre la sous-alimentation, notamment infantile, et seront obérés par l'accroissement démographique.

11 Nsombo, B., Thomas, T. et al., East African Agriculture and Climate Change. A Comprehensive Analysis - DRC, décembre 2012, IFRPI/ CGIAR/ASARECA, 2p. 


\section{Tanzanie}

En Tanzanie, où près des $3 / 4$ de la population vit de la terre, l'agriculture et la sécurité alimentaire constituent le cœur de la lutte contre la pauvreté. Même si la part de l'agriculture dans le PIB décroit progressivement

Hormis la moitié Est de la Tanzanie, qui pourrait connaitre de légères augmentations des précipitations, le volume des pluies devrait rester stable sur la majeure partie du territoire à l'horizon 2050. Les modèles convergent vers une hausse des températures, autour de $1^{\circ} \mathrm{C}$ pour deux des quatre modèles appliqués à l'Afrique de $1^{\prime}$ 'Est par 1'IFPRI, et de $2^{\circ} \mathrm{C}$ et plus pour les deux autres.

La prévalence de la malaria et du choléra est liée à des températures élevées et de fortes précipitations (certaines parties ouest de la Tanzanie dépassent déjà les 2000 mm de pluie par an).

L'application du modèle climatique MIROC à la culture sous pluie du maïs conduit selon les zones à des gains et à des pertes de rendement oscillant entre plus et moins $25 \%$ (2050 comparé à 2000, cf. tableau n²4).

Outre la différence entre les modèles, on constate que des projections nationales n'ont qu'un intérêt limité, tant les différences sont marquées localement.

En revanche, le doublement probable de la population d'ici à 2050 exacerbera la pression sur ressources renouvelables (eau, sols pour l'agriculture,...). Or la production de maïs ne suivra pas l'augmentation de la population, ce qui signifie des importations en hausse, ainsi que le prix de vente de cette denrée. A l'inverse, le sorgho devrait bénéficier de l'accroissement des températures avec de meilleurs rendements et une surface étendue dédiée à cette culture. Cela conduirait au quadruplement de la production de sorgho d'ici 2050, avec des perspectives d'exportation, avec un prix de vente sur les marchés internationaux en hausse, quels que soient les scénarios de population et de croissance économique.

\section{Autres conséquences du changement climatique en Afrique de l'Est et RDC}

Outre ses effets sur la santé (malnutrition, augmentation de la prévalence de maladies comme le choléra ou la malaria, épidémies touchant les cheptels ou les cultures) évoqués dans la partie consacrée à l'agriculture, le réchauffement de l'Afrique de l'Est va également entrainer des conséquences dans d'autres domaines essentiels.

\section{Mouvement de population et urbanisation accrue}

Le seul lien incontestable entre migration et dégradation de l'environnement concerne les évènements climatiques extrêmes, qui favorisent une mobilité d'urgence, le plus souvent au sein du même Etat (ou des pays limitrophes). En revanche, le changement 
climatique (sécheresse en particulier) va également favoriser des mouvements lents et progressifs de population, liés à la dégradation des conditions de vie en milieu rural. Ces migrations sont alors tout autant économiques qu'environnementales. La décision de départ, plus individuelle que collective, se fait sur des critères de détérioration des revenus et de survie. En ce sens, l'urbanisation peut être vue comme une stratégie d'adaptation, et les zones urbaines offrent davantage d'opportunités pour construire des communautés plus résilientes au changement climatique. En revanche, les populations urbaines défavorisées sont les plus exposées aux crises alimentaires (accessibilité financière, disponibilité, stabilité).

\section{Ressources en eau}

Les modélisations de l'IFPRI ne différencient pas assez « l'eau bleue », qui constitue les flux et stocks des aquifères, des lacs, des cours d'eau utilisés pour les usages humains, et « l'eau verte », présente dans les sols, indispensable aux cultures sous pluie et aux écosystèmes. L'augmentation des précipitations risque d'accroître le ruissellement, donc les flux d'eau de surface (et le risque d'inondation). Elle pourrait ne bénéficier que de manière secondaire à l'humidité présente dans les sols, d'autant que la hausse des températures dans toute la zone d'étude va accroître l'évapotranspiration. Ainsi, malgré des modélisations conduisant à des précipitations moyennes plus élevées ${ }^{13}$, l'eau effectivement utile pour l'agriculture sous pluie et les écosystèmes pourrait décroitre (certaines parties du Kenya et de la Tanzanie).

\section{Impacts sur les infrastructures ${ }^{14}$ (niveau des mers, évènements climatiques extrêmes,...)}

Les infrastructures en zone littorale sont menacées par la hausse du niveau des mers, qui pourrait atteindre $70 \mathrm{~cm}$ d'ici 2070. Plus que la submersion, les évènements extrêmes tels les cyclones constituent le risque majeur rattaché à l'élévation du niveau des mers. En effet, cette dernière étend l'aire de vulnérabilité aux évènements extrêmes. Ainsi, des cyclones toucheront des zones et des infrastructures qui n'étaient pas auparavant exposées à ces risques. Des dommages à la zone portuaire de Dar es Salam pourraient avoir de fortes conséquences économiques en Tanzanie (95\% du commerce international du pays y transite) et sur les approvisionnements des pays enclavés des Grands Lacs. Les complexes touristiques de Mombasa se situent sur le littoral, et sont exposés à la montée des océans.

Les évènements extrêmes menacent également la continuité de services essentiels (eau, électricité, santé...), à l'image des crues du fleuve Tana en 2009 au Kenya qui avaient privé de soins médicaux plus de 100000 personnes.

13 IPCC, «Climate change and water », Technical paper n ${ }^{\circ}$ IV, 2008, disponible sur le site internet du GIEC : www.ipcc.ch 14 World Bank, $4^{\circ}$. Turn Down the Heat. Climate Extremes, Regional Impacts, and the Case of Resilience, Washington DC, World Bank, June 2013, 213p. 


\section{Production d'énergie}

Les conséquences du changement climatique sur la production hydroélectrique en Afrique n'ont fait l'objet que de rares travaux, en particulier sur les bassins fluviaux situés dans les régions subhumides. A titre d'exemple, des simulations du changement climatique pour la station hydroélectrique de Batoka Gorge sur le fleuve Zambèze ${ }^{15}$ prévoient à l'horizon 2080 une évolution significative du régime fluvial (avec une diminution du débit mensuel moyen de 3,21 km3 à 2,07 km3) et une réduction de la production d'électricité (diminution de la production mensuelle moyenne de $780 \mathrm{GWh}$ à $613 \mathrm{GWh}$ ). Dans la région des Grands Lacs, le changement climatique compliquera l'exploitation et le fonctionnement optimal des barrages hydroélectriques. A cause de fortes sécheresses en 2005/2006, la production d'hydroélectricité tanzanienne a été divisée par deux. Ces mêmes années, la baisse du niveau du Lac Victoria (-1,5m) a diminué de $40 \%$ la capacité de production de 1'Uganda Electricity Generation Company. Des pluies plus concentrées ne pourront pas forcément être stockées dans un lac-réservoir, et ne compenseront donc pas l'allongement du nombre de jours sans pluie consécutifs.

Les pays couverts par l'Observatoire des Grands Lacs révèlent une extrême vulnérabilité climatique en matière de production d'énergie. La majorité de l'électricité kenyane provient de barrages dans le bassin du fleuve Tana. De fortes sécheresses à l'instar de celles de 2011, sans précédent depuis une soixantaine d'années, menacent directement la fourniture d'électricité dans la quasi-totalité du territoire ${ }^{16}$. De même, le niveau des lacs Kivu et Tanganyika aura un impact direct sur la capacité de production d'électricité au Rwanda, dans l'est de la RDC et au Burundi.

La multiplication des projets hydroélectriques, s'ils se concrétisent, pourrait conduire à des modifications des débits des cours d'eau, soit en les différant dans le temps (entre saisons, voire d'une année sur l'autre), soit en les réduisant (évaporation due aux lacs de retenue, réservoir servant également à des projets d'irrigation,...). Si les barrages contribuent à amortir les effets des épisodes climatiques extrêmes (utilité des lacs de retenue en période sèche, lissage des inondations), ils ont des impacts sociaux et environnementaux. Les projets hydroélectriques éthiopiens sur l'Omo pourraient ainsi modifier durablement l'environnement naturel et humain du lac Turkana, dont les caractéristiques (alcalin, salinité) le rendent particulièrement vulnérables à des variations quantitatives de ses sources.

De plus, le changement climatique accentuera les conflits entre les usages énergétiques et agricoles de l'eau. Le calendrier des lâchers d'eau pour produire de l'hydroélectricité pourra ne pas coïncider avec le calendrier des besoins en eau pour l'agriculture. Les arbitrages politiques pour la répartition de la ressource en eau (entre énergie et agriculture principalement) s'annoncent ainsi plus complexes et plus fréquents. Cette concurrence entre usages de l'eau se déroulera à l'échelle interne ou régionale.

15 Harrison, G. P. \& Whittington, H. W. "Susceptibility of the Batoka Gorge hydroelectric scheme to climate change", Journal of Hydrology, 264 (1-4), juillet 2002, 230-241.

16 ACCES (Africa, Climate Change, Environment and Security), Climate Change and Security in Africa. Vulnerability Discussion Paper, Africa - UE Partnership, octobre 2010, 65p. 
Le fonctionnement des centrales thermiques, dont le refroidissement dépend d'importants volumes d'eau à une température maximale donnée, pourrait également être affecté à la fois par la baisse des débits d'étiage et par la hausse des températures (ce qui aura une incidence sur la température des cours d'eau). Les perspectives de croissance de l'usage des hydrocarbures (électricité, chaleur) dans la région pourraient être limitées, au moins de manière saisonnière ou plus ponctuelle, par les conditions climatiques.

Au regard de l'accroissement démographique en Afrique de l'Est et au Congo, l'usage quasi généralisé de la biomasse pour l'énergie accentuera la déforestation (particulièrement en Ouganda, au Kenya, et au Burundi).

\section{La priorité : l'adaptation de l'agriculture au changement climatique}

L'agriculture en l'Afrique de l'Est et RDC revêt une dimension sociale primordiale, pilier de la (sur-)vie quotidienne de la moitié (RDC) voire des trois-quarts de la population des différents États (Kenya, Rwanda, Ouganda, Tanzanie) de la zone d'étude.

Dès lors, la priorité parmi les actions d'adaptation au changement climatique doit être donnée au soutien et à la transformation du secteur agricole dans cette zone. Le renforcement de la sécurité alimentaire passe par la réduction des vulnérabilités agricoles aux évolutions climatiques.

La principale vulnérabilité de l'agriculture des pays des Grands Lacs vient tout d'abord du fait que la culture se pratique essentiellement sous pluie, le recours à l'irrigation étant l'exception. Le développement de l'irrigation semble notamment approprié dans les zones arides et semi-arides du Kenya ou de la Tanzanie, dans les limites d'une durabilité économique (coût énergétique du pompage dans les eaux souterraines par exemple) et environnementale (pression supplémentaire sur la ressource en eau dans des zones sèches...).

Le contrôle de l'accroissement démographique réduira également les vulnérabilités de la sécurité alimentaire. En effet, l'amélioration attendue des rendements agricoles, grâce à l'augmentation des températures et des précipitations dans les quarante prochaines années, devrait être estompée par la croissance de la population.

Les incertitudes reflétées par les modèles climatiques dans la sous-partie précédente incitent à adopter d'ores et déjà des «no-regret policies $»^{17}$. Ces politiques « zéro regret » auront des bénéfices sur la prévention et la réduction des risques environnementaux, même si le réchauffement global s'avère moins élevé que prévu. Des actions visant par exemple à l'amélioration des réseaux routiers, des infrastructures et des plateformes d'échanges alimentaires renforceront la sécurité alimentaire de cette zone, quelle que soit l'intensité des changements climatiques à venir. Elles amélioreraient le commerce de denrées, entre les zones favorisées par les changements climatiques (rendements agricoles accrus...) et celles souffrant d'une aridité croissante. La réforme des régimes fonciers s'inscrirait également dans cette démarche, en responsabilisant et impliquant davantage les fermiers dans la gestion pérenne des terres qu'ils exploitent.

17 A ce sujet, cf. Busby, J. W. Climate Change and National Security. An Agenda for Action, New York, Council on Foreign Relations, $\operatorname{CSR} n^{\circ} 32$, novembre 2002, 32p. 
Même si les conditions climatiques moyennes (principalement températures et précipitations) ne changent pas radicalement d'ici à 2050, la variabilité interannuelle devrait s'amplifier avec des exacerbations passagères des caractéristiques climatiques et davantage d'évènements extrêmes. Le soutien à la mise en culture de variétés végétales plus résistantes à la chaleur et à des conditions climatiques plus contrastées, à l'image du sorgho.

Le soutien à la recherche semble enfin primordial, pour soutenir l'augmentation des rendements, et prévenir les maladies et épidémies, végétales et humaines, qui accompagneront le réchauffement de la région.

\section{Changement climatique et conflictualité en Afrique de l'Est}

Une relation positive entre climat et conflictualité a été trouvée dans les régions arides et semi-arides d'Afrique de l'Est, avec comme facteur marginal des conflits des volumes anormaux de précipitation ${ }^{18}$, qu'il s'agisse de périodes sèches ou au contraire d'inondations. Mais cette relation n'est valide qu'à l'échelle interne (à partir de référencements de violences à l'échelle sub-nationale), et non internationale. Des pluies anormalement élevées favoriseraient des tensions communautaires, tandis que des sécheresses augmenteraient les évènements violents impliquant des groupes rebelles (en l'occurrence la LRA en Ouganda et dans les pays limitrophes).

D'autres publications suggèrent une augmentation de la conflictualité les années suivant des épisodes de pluie plus abondante que la moyenne ${ }^{19}$. A l'opposé, les années postérieures à une forte sécheresse seraient plus paisibles. Cela renforcerait l'hypothèse que les violences à grande échelle entre groupes/communautés découlent davantage de calculs et de tentatives de gains politiques que de comportements désespérés pour de l'eau, des terres ou des pâturages. Les enjeux environnementaux font fréquemment l'objet d'une instrumentalisation politique, par le biais par exemple d'une ethnicisation d'enjeux tels que l'accès à l'eau, aux terres communautaires, des revendications relatives aux terres, aux droits de migration et de passage... Les années électorales font d'ailleurs l'objet de davantage de violences. Dès lors, la part causale de l'environnement dans des conflits et violences apparait comme difficile à isoler, tant elle s'inscrit dans des contextes sociaux, communautaires et politiques.

18 Raleigh, C. \& Kniveton, D. "Come rain or shine: An analysis of conflict and climate variability in East Africa", Journal of Peace Research, janvier 2012, 49 (1), pp. 51-64

19 Theisen, O. M. "Climate clashes? Weather variability, land pressure, and organized violence in Kenya, 19892004”, Journal of Peace Research, janvier 2012, 49 (1), pp. 81-96. 
Une ambitieuse publication ${ }^{20}$ parue en 2012, et fondée sur 16359 évènements violents en Afrique de l'Est entre 1990 et 2009, conforte la complexité d'isoler les évolutions de l'environnement comme facteur d'instabilité. L'étude souligne l'existence d'un lien d'un lien entre des températures élevées et le risque d'évènements violents. Une augmentation modérée des températures réduit à l'inverse la probabilité de conflits. Si ce lien semble mesurable, l'article conclut que les facteurs socioéconomiques, politiques et géographiques jouent un rôle bien plus substantiel dans l'occurrence de conflits que le changement climatique en Afrique de l'Est.

Face à la complexité d'un objet social tel que le conflit, sans doute le biais méthodologique originel est-il la recherche d'un lien de causalité quantifiable entre conflits et dégradations environnementales. Les corrélations mises en lumière ne valent pas explications, et ne sont pas étayées par des modèles théoriques. Le rapprochement entre conflictualité et évolutions de l'environnement fait l'objet d'études depuis la fin des années 1980 par différentes écoles de pensée, à Toronto (Thomas Homer-Dixon), en Suisse (Günter Baechler), et plus récemment à Olso (autour de Peter Gledtisch), ou à Washington DC (Geoffrey Dabelko). Si ces travaux ont été redynamisés par la progression de la recherche sur le changement climatique, le lien de causalité entre dégradations environnementales et conflits ou tensions demeure très discutée ${ }^{21}$. Il s'agit toujours de travaux a posteriori, à la valeur prédictive incertaine, et aux conclusions très générales (du type « une relation positive entre changement climatique et conflits a été identifiée $» . .$.$) . Cette abondante littérature grise s'appuie généralement sur des zones géographiques où$ les tensions, jusqu'aux guerres civiles et interétatiques, ont été fréquentes. Le dossier « Climate Change \& Conflict » du Journal of Peace Research de janvier 2012 comprend en ce sens sept articles consacrés à l'Afrique (dont quatre sur l'Afrique de l'Est ou le Kenya ${ }^{22}$ ) sur les neuf traitant d'exemples régionaux.

L'impossibilité de quantifier la part du changement climatique comme source de conflit ne signifie pas pour autant que ce lien n'existe pas. Il convient de substituer à la recherche d'une causalité, des approches par les risques et de l'identification des vulnérabilités. Cela passe par la réintégration des enjeux climatiques et environnementaux dans la complexité des dynamiques sociales, économiques et politiques, aux différentes échelles de l'analyse.

20 O'Loughlin, J., Witmer, F. et al. “Climate variability and conflict risk in East Africa, 1990-2009”, PNAS 2012 ; published ahead of print October 22, 2012, doi:10.1073/pnas.1205130109

21 Voir par exemple Tobias, H., « Confronting the Concept of Environmentally Induced Conflict», Peace, Conflict and Development, Bradford, vol. 6, janvier 2005.

22 Hendrix, C. S. \& Salehyan, I. «Climate change, rainfall, and social conflict in Africa », Journal of Peace Research, janvier 2012, 49 (1), pp. 35-50; Gleditsch, N. P. "Whither the weather? Climate change and conflict", Journal of Peace Research, janvier 2012, 49 (1), pp. 3-9; Adano W.R., Dietz, T. et al. "Climate change, violente conflict and local institutions in Kenya's drylands", Journal of Peace Research, January 2012, 49 (1), pp. 65-80; Raleigh, C. \& Kniveton, D., op.cit.; Theisen, O. M., op.cit.; Butler, C. K. \& Gates, S. "African range wars: Climate, conflict, and property rights", Journal of Peace Research, janvier 2012, 49 (1), pp. 23-34; Busby, J., Smith, T.G. et al. "Climate change and insecurity. Mapping Vulnerability in Africa”, International Security, vol. 37, n$^{\circ} 4$, été 2013 , pp. 132-172. 


\section{Conclusion}

Les extrapolations des modèles climatiques globaux à l'échelle des pays des Grands Lacs (et en Tanzanie et au Kenya) conduisent à des incertitudes et divergences de résultats. Pour devenir un véritable outil d'aide à la décision, l'identification des risques climatiques requiert des scénarios climatiques plus robustes ${ }^{23}$, étayés par des modélisations du climat à la résolution affinée (entre 90 et $100 \mathrm{~km}$ dans les travaux actuels). Ces divergences nourrissent la vision optimiste d'une Afrique des Grands Lacs recevant plus de précipitations dans les prochaines décennies, et bénéficiant au final du réchauffement global. Cette perception doit être minorée en premier lieu par le fait que les évènements climatiques seront plus marqués (pluies plus concentrées et plus espacées, accentuant à la fois les risques d'inondation et de sécheresse), et que l'aridité des zones déjà sèches pourrait s'accroître (large bande de territoire de la frontière tanzano-kenyane à la frontière soudanoéthiopienne). Ensuite, les nombreuses vulnérabilités aux changements environnementaux, aggravées par l'accroissement démographique, sont multisectorielles et multidimensionnelles, menaçant l'agriculture, la production d'énergie, les infrastructures, la santé, les ressource en eau, avec des impacts sociaux, économiques et politiques. La sécurité alimentaire et la production d'électricité apparaissent comme les deux domaines les plus impactés par le changement climatique en Afrique de l'Est.

Si l'existence de liens entre changement climatique et conflictualité dans cette aire géographique se consolide, la part de la dégradation de l'environnement dans les conflits semble accessoire en comparaison de facteurs socioéconomiques, politiques et géographiques. Une instrumentalisation politique croissante des enjeux environnementaux (terres, eau, droits de passage,...), notamment par leur ethnicisation, pourrait être l'effet indirect du réchauffement global qui aurait le plus d'impacts sécuritaires en Afrique de l'Est.

23 Wanneau, K. \& Gemenne, F. Climate and Security. Evidence, Emerging Risks and a New Research Agenda, IDDRI, Working paper, n¹2/13, juillet 2013, 22p., http://www.iddri.org/ Publications/Collections/ Idees-pour-le-debat/WP1213_FG\%20KW_ climate $\% 20$ and $\% 20$ security.pdf 